\title{
Vom gemessenen zum angemessenen Körper - Human Enhancement als historischer Prozess
}

\section{Iris Ritzmann}

Medizinhistorisches Institut und Museum, Universität Zürich

\section{* Die Literaturangaben finden sich unter www.saez.ch $\rightarrow$ Aktuelle Nummer oder $\rightarrow$ Archiv $\rightarrow 2013 \rightarrow 11$.}

Korrespondenz:

Schweizerische Akademie der Medizinischen

Wissenschaften (SAMW)

Petersplatz 13

CH-4051 Basel

mail[at]samw.ch
In der Diskussion um die normative Einordnung von Angeboten des Human Enhancements wird häufig versucht, Grenzen zwischen medizinisch vertretbaren und gesundheitsschädigenden, zwischen wissenschaftlich gesicherten und unseriösen, letztlich zwischen moralisch zulässigen und verwerflichen Eingriffen zu ziehen. Die heutigen Ansichten, wo diese Grenzen verlaufen sollen, sind jedoch das Resultat von Diskursen, die sich über längere Zeiträume erstrecken und vom soziokulturellen Umfeld der jeweiligen Epoche geprägt sind.

Dieser historische Beitrag setzt sich deshalb nicht zum Ziel, einen Überblick über die Geschichte des Human Enhancements von der Antike bis heute zu leisten. Er will vielmehr anhand punktueller Darstellungen dazu beitragen, die Relativität heutiger Sichtweisen vor Augen zu führen und zugleich Entwicklungsprozesse durch die zeitliche Dimension sichtbar werden zu lassen.

Für die Entstehungsgeschichte des Human Enhancements sind Prozesse auf unterschiedlichen Ebenen relevant, die in diesem Beitrag nur ganz oberflächlich angesprochen werden können: auf gesellschaftlicher Ebene die Verbreitung medizinischer Denkweisen und die Etablierung eines menschlichen Norm- und Idealbilds, auf ökonomischer Ebene Veränderungen auf dem medizinischen Markt und damit Umstrukturierungen in der Arzt-Patienten-Beziehung und auf individueller Ebene resultierende Ängste und Wünsche in einem vermehrt an Perfektion und Leistung orientierten Umfeld.

\section{Medikalisierung der Gesellschaft seit der «Aufklärung"}

Historische Abhandlungen über Human Enhancement setzen häufig mit der Antike ein, um dann über das Mittelalter und die Frühe Neuzeit mit Belegen aufzuwarten, dass Menschen «have always been interested in creating or bettering themselves» [1]*. Ob und inwiefern diese Verhaltensweise nun wirklich eine biologische Konstanz aufweist, scheint zur Analyse der heutigen Situation weniger beizutragen als die Untersuchung, wie diese Verbesserungswünsche von medizinischen Anbietern aufgegriffen wurden, wie sie in unterschiedlichen Kreisen diskutiert und instrumentalisiert wurden und welche Wirkungen von ihnen ausgingen.

Aufgrund dieses Fragespektrums liegt es nahe, die historische Analyse mit dem späten 18.Jahrhundert zu beginnen. In dieser Epoche wächst die medizi-
«Human Enhancement» bezeichnet medizinische oder biotechnologische Interventionen, deren Zielsetzung nicht primär therapeutischer oder präventiver Art, sondern eine «Verbesserung» nichtpathologischer Merkmale ist. Eine Arbeitsgruppe der Akademien der Medizinischen Wissenschaften (SAMW) und der Geistes- und Sozialwissenschaften (SAGW) hat die ethischen Fragen des Enhancements vertieft untersucht und ihre Analysen und Empfehlungen in der Broschüre «Medizin für Gesunde?» (www.akademien-schweiz.ch $\rightarrow$ Projekte und Themen) veröffentlicht. In loser Folge erscheinen in der SÄZ Auszüge aus dem Bericht.

nische Vereinnahmung menschlicher Verbesserungswünsche. Ebenso entstanden damals die Grundzüge eines staatlichen Gesundheitswesens, das die Gesundheit des einzelnen Individuums im gesellschaftlichen Rahmen definierte [2].

Wir leben heute in einer Gesellschaft, deren Werte und tägliche Umgangsformen in hohem Masse von medizinischen Denkstilen geprägt sind. Die Medizingeschichte nennt dieses Phänomen «Medikalisierung». Medizinische Interpretationen bestimmen mit, was wir essen, wie wir unsere Freizeit gestalten, welche Feriendestinationen wir wählen. Dieser «innere Arzt», der die individuelle und einzigartige Sichtweise einzelner Personen verkörpert, steht aber nicht nur in engster Beziehung zur Erfahrungswelt der Betreffenden, sondern immer auch zum breiteren gesellschaftlichen Verständnis von Gesundheit und damit zur Medizingeschichte.

Im Zeitalter der sogenannten Aufklärung richteten paternalistische Staatsgebilde aus politischem, militärischem und wirtschaftlichem Interesse ihr Augenmerk auf die Gesundheit ihrer Bürgerinnen und Bürger, auf möglichst zahlreiche Geburten ge-

\section{Mitglieder der Arbeitsgruppe}

Prof. Dr. med. Dr. phil. Nikola Biller-Andorno (Vorsitz)

Prof. Dr. med. Anne-Françoise Allaz

Dr. phil. Gaia Barazzetti (bis 2010)

lic. ès lettres Nadja Birbaumer, SAGW

PhD Susanne Brauer, NEK-CNE

Prof. Dr. med. Jürg Kesselring

Prof. Dr. med. lic. phil. Iris Ritzmann

Dr. phil. Simone Romagnoli

Dr. biol. Adrian Rüegsegger, TA-SWISS

lic. iur. Michelle Salathé, MAE, SAMW

PD Dr. theol. Markus Zimmermann-Acklin 
sunder Kinder, auf arbeitskräftige und fruchtbare Erwachsene und auf die Vermeidung von Armut, Krankheiten und Behinderungen. Der zeitgenössische Begriff der «Medicinischen Polizey» - am bekanntesten wurde er über das von 1779 bis 1819 publizierte sechsbändige Werk «Vollständiges System einer medicinischen Polizey» des Mediziners Johann Peter Frank stand für die Vorstellung einer Gesundheitsfürsorge, die reglementierend in das Verhalten der Bevölkerung eingreift und die Menschen «von der Wiege bis zur Bahre» umfassend begleitet.

\section{Die Etablierung einer medizinischen Expertenkultur wirkte sich sowohl auf das Heilangebot wie auch auf die Wahrnehmung von Körperlichkeit aus.}

Im Allgemeinen wird die medizinische Tätigkeit im 18. Jahrhundert mit zunehmenden ärztlichen Bemühungen zur Hebung der «Volksgesundheit» in eine enge Beziehung gebracht. Die Schaffung staatlicher Fürsorgeeinrichtungen und der Kampf gegen nichtapprobierte Heiler, aber auch eine gesteigerte publizistische Aktivität einzelner Ärzte charakterisieren dieses Verständnis der «Aufklärungsmedizin». Gesundheit wurde ein gesellschaftliches Gut, das es zu schützen und zu erhalten gilt.

Die populärmedizinische Ratgeberliteratur erlebte einen deutlichen Aufschwung; die medizinischen Werke richteten sich in der Landessprache an die breite Bevölkerung, auch an die Bewohner ländlicher und abgelegener Regionen, soweit sie des Lesens mächtig waren. In Titel und Vorwort hoben die schreibenden Ärzte deutlich hervor, dass sie die Gesellschaft über Gesundheit und Krankheit belehren und aufklären wollten. Zahlreiche Zeitschriften beschäftigten sich in der zweiten Hälfte des 18.Jahrhunderts mit medizinischen und gesundheitserzieherischen Fragen. Bei einem wachsenden Anteil lesender Bevölkerungsteile gelang es unterschiedlichen Medizinern, darunter vor allem vielen Pietisten, über das Medium der Publikationen ihre Gesundheitsvorstellungen zu verbreiten und zu zementieren.

\section{Arztberuf zwischen Dienstleistung und Fachautorität}

«Die Beachtung der Menschenwürde und der Respekt vor der Selbstbestimmung des Patienten sind die Grundlage jeglichen ärztlichen Handelns» [3], formulierte der Expertenbericht der SAMW zur Zukunft der Medizin im 21.Jahrhundert. Trotz gegenläufiger Entwicklungen ist die Arzt-Patienten-Beziehung heute noch mehrheitlich auf der ärztlichen Seite von Wissen und Macht, auf der Seite der Patienten von Hilfsbedürftigkeit und Unselbständigkeit gekennzeichnet. Hier müsste also die Mündigkeit der $\mathrm{Pa}$ - tienten gefördert werden, was jedoch den Widerstand von unterschiedlicher Seite hervorruft. Inwiefern dürfen Patienten Ansprüche stellen und das Behandlungsangebot nach eigenen Wünschen nutzen? Antworten auf diese Fragen betreffen auch die Auseinandersetzung mit den Marktangeboten des Human Enhancements; sie berühren finanzielle Aspekte und ganz zentral das Rollenverständnis von Ärztin bzw. Arzt und Patient bzw. Patientin, das es zu analysieren gilt.

Die Ärzte der frühen Neuzeit besassen noch kein Monopol auf dem medizinischen Markt. Sie mussten ihre Position gegen andere handwerkliche und freie Anbieter verteidigen. Den Patienten gegenüber befanden sie sich in einer weitestgehenden finanziellen Abhängigkeit. Wie die ärztliche Ratgeberliteratur des 18. Jahrhunderts verrät, sollte sich ein Arzt eine Stammkundschaft zulegen, die sich in gesunden und kranken Tagen vertrauensvoll an ihn wendet. Weitgehend unabhängig von der therapeutischen Qualität galt es, die Klientel zu überzeugen, dass sie bei ihm, und nur bei ihm, ihre Gesundheit erhalten oder wiedererlangen könne.

Die Stammkundschaft des gelehrten Arztes bestand im 18. Jahrhundert in der Regel aus einer eher überschaubaren Gruppe Privatpatienten, von deren Zahlungsfreudigkeit das Einkommen des Arztes letztlich abhing. Die Medizingeschichtsschreibung bezeichnet diese Beziehungsform zwischen Arzt und Patient als «Patronagesystem» ${ }^{*}$. Dem Patienten kommt in diesem Modell, das vor allem im 17. und 18. Jahrhundert zum Tragen kam, die Rolle des Patrons zu, um dessen Gunst der gelehrte Arzt buhlte. Auch wenn einzelne Ärzte zuweilen finanziell unabhängig waren, so musste sich doch die grosse Mehrheit in dieser Marktlage eine Existenz sichern.

Die Abgrenzung gegenüber anderen Anbietern stand daher ganz im Zentrum der medizinischen Tätigkeit. Erst im Verlauf des 19. Jahrhunderts erlangten die akademischen Ärzte eine gewisse Monopolstellung auf dem medizinischen Markt und damit die relative Definitionsmacht über die Begriffe gesund und krank, gefährlich und risikoarm, wirksam und obsolet. Sie bestimmten immer eigenständiger mit, wessen Heiltätigkeit anerkannt wurde und gegen wen die Obrigkeit vorgehen sollte, notabene ohne dass sie Krankheiten besser als ihre Konkurrenz zu heilen vermochten. Das pluralistische Angebot verringerte sich zusehends, und nichtakademische Heilkundige wurden als unseriöse Heiler abgewertet. Demgegenüber wuchs das Vertrauen gegenüber den studierten Ärzten, das bis heute die Basis der ArztPatienten-Beziehung bildete und die Selbständigkeit der Kranken weitgehend untergrub.

Dieser Aufstieg der akademischen Ärzteschaft erfolgte in erster Linie über die Etablierung eines neuen Gesundheitswesens. Er hatte mehr mit der fortschreitenden Medikalisierung der Gesellschaft als mit den zur Verfügung stehenden Therapien zu tun. In diesem Kontext konnten sich Ärzte als Experten situieren, 
die bestimmten, welche Gefahren der Bevölkerung drohen und womit sie bekämpft werden sollen. Mit den aufkommenden naturwissenschaftlichen Lehren erwuchs den akademischen Medizinern zudem eine Argumentationsbasis, die nicht eigene Konzepte gegen andere Konzepte stellte, sondern die eine objektive und unabhängige Wissenschaft vertrat.

\section{Kollektivierte Gesundheit}

Mit den kollektiven Aufgaben der Medizin veränderte sich die persönliche Arzt-Patienten-Beziehung. Ärzte waren nicht allein für einzelne Kranke, sondern für die Gesundheit der ganzen Bevölkerung zuständig. Die «Volksgesundheit» vor drohenden Gefahren zu beschützen, wurde Aufgabe der Medizin.

Auch auf Patientenseite trug der Einzelne Verantwortung für die eigene Gesundheit und darüber hinaus für die Gesundheit der ganzen Bevölkerung. Gesunderhaltung war nicht nur Bedingung für ein möglichst arbeitsintensives, reproduktives Leben des Einzelnen, sondern auch für Wehrkräftigkeit, Leistungskraft und Wohlstand des ganzen «Volkes».

Die Abwendung möglicher Bedrohungen verhalf einer Präventivmedizin zum Aufstieg, deren Angebote sich primär an gesunde Personen richteten, sei es im grösseren gesellschaftlichen Umfeld oder auf der Mikroebene des einzelnen Individuums. Medizinische Bedrohungsszenarien müssen freilich zuerst explizit propagiert werden, bevor sie allgemein Anerkennung finden. Diese Strategien erklären sich nicht einfach aus den historischen Fakten heraus. Nach der breiteren Rezeption von Ludwik Flecks Theorien hat sich heute die Sichtweise durchgesetzt, dass es weder «die» Medizin als einheitliches, stetig vorwärts strebendes und in sich logisches System noch eine unabhängige Wissenschaft gibt.

Ärzte definierten, was Gesundheit beinhaltete, welche Gefahren ihr drohten und wie sich diese Gefahren vermindern liessen. Zum Beispiel identifizierten Aufklärungsärzte des 18.Jahrhunderts die Onanie als schreckliches Übel, das die gesamte junge Generation bedrohte. Im 19. Jahrhundert erkannten sie in der verminderten Reproduktion der Bevölkerung eine neue Gefahr, die es abzuwenden galt. In den 1880er Jahren gerieten Alkoholismus und Kriminalität ins ärztliche Visier, und wenige Jahrzehnte später sollte mit eugenischen Massnahmen eine drohende Degeneration der Zivilvölker bekämpft werden. Die damals moderne und zukunftsorientierte, von Ärzten aller westlichen Nationen propagierte «Wissenschaft» der Erb- und Rassenkunde lehrte, dass ein gesundheitszersetzender, lebensbedrohlicher Einfluss von Trägern fremden Erbguts auf die restliche Bevölkerung ausging. Der Wille zur Unfruchtbarmachung und sogar Ausmerzung angeblich normabweichender Menschen basierte nicht zuletzt auf einer Angst vor Degeneration und Untergang.

Normierung von Gesundheit und Krankheit

Die Etablierung einer medizinischen Expertenkultur wirkte sich sowohl auf das Heilangebot wie auch auf die Wahrnehmung von Körperlichkeit aus. Ein modernes Gesundheitssystem, das eine wachsende Bevölkerung versorgen sollte, bedurfte messbarer Kriterien, um Krankheit von Gesundheit zu unterscheiden und allgemeine Richtlinien zu erlassen. Die ursprünglich antike Lehre der Humoralpathologie, die das individuelle Säftegleichgewicht für die Gesundheit verantwortlich machte und jede Krankheit mit der Persönlichkeit des Patienten und seinen Lebensgewohnheiten erklärte, wich einer Systematisierung, die Krankheit einzelnen Kategorien zuordnete. Diese Klassifizierung der Krankheiten bedingte geradezu, sich an Normen zu orientieren.

Diagnostik und Therapie, aber auch ganz allgemein Gesundheit und Krankheit verloren ihre Individualität. Thermometer, Pulszählung, Atmungsfrequenz und schliesslich die Sichtbarmachung von elektrischen Organtätigkeiten veränderten die Körperwahrnehmung. Gesund war nicht mehr, wer sich gesund fühlte, sondern beispielsweise, dessen Körpertemperatur zwischen 35,5 und $37^{\circ} \mathrm{C}$ betrug. Die Visualisierung des Körperinnern, von der Röntgentechnik über den Ultraschall bis zu CT und MRI, ermöglichte die Erkennung von immer neuen, noch detaillierteren Normabweichungen. Röntgenbilder legten den Schweregrad der Lungeninfektion fest, Ultraschallbilder urteilten über die Normalität der Schwangerschaft und des Ungeborenen, oder ein MRI deckte eine bisher unentdeckte Veränderung auf.

Bilder, Tabellenwerte, Blut- und schliesslich Genanalysen liessen neue Formen von Patienten entstehen, Patienten, die eigentlich keine Kranken waren und dennoch nicht der Norm entsprachen. Der Anteil an «normalen» Individuen schwand mit den zunehmenden technischen Möglichkeiten der Diagnostik. Eine wachsende Gruppe Menschen, die beispielsweise durch eine Heruntersetzung der Blutdrucknormwerte oder neue genetische Analysen zu potentiellen Patienten wurden, fühlte sich in ihrem Alltag von Krankheit und Tod bedroht und wurde zu regelmässigen Nutzern medizinischer Angebote.

Normvorstellungen des Körpers dominierten nicht nur den medizinischen Fachdiskurs; sie sprangen auf die Wahrnehmung von Krankheit und Gesundheit breiter bürgerlicher Kreise über. In einer vermehrt auf das Kollektiv ausgerichteten Gesellschaft gewann die Orientierung an Normen an Bedeutung der «innere Arzt» drang verstärkt ins Alltagsverhalten vor.

\section{Enhancement im Zeichen idealisierter Normen}

Norm entspricht nicht einfach einem definierten Abschnitt einer Gauss'schen Normalverteilung. Norm heisst auch, wie beispielsweise die Lehrbücher der Anatomie und andere visuelle Zeugnisse des Körperverständnisses verraten, jung, schön und in vielen Fällen männlich. Die normale und damit «gesunde» Haut ist bis in die heutigen Darstellungen eine Haut 
ohne Falten und Flecken, der normale Bauch flach, die normale Nase gerade und die normalen Zähne weiss und ebenmässig. Auch das normale, gesunde Körpergewicht entsprach nicht mehr dem Durchschnittsgewicht, sondern gewann an Eigendynamik, sodass plötzlich die eigentlich Norm als abnorm, ungesund und pathologisch zu gelten begann. Diese veränderte Norm repräsentierte nicht nur Gesundheit, sie wurde zum Ideal.

Wollte man dieser idealisierten Norm entsprechen, also als normal und gesund wahrgenommen werden, mussten Körper und Geist entsprechend geformt sein. Die Nachfrage nach Angleichung des eigenen Ichs an Idealvorstellungen, die von Ärzten mitgeprägt wurden, traf auf entsprechende Angebote, deren Spektrum sich mit den Möglichkeiten der Medizin zusehends vergrösserte. Human Enhancement wurde zu einem attraktiven Einkommenszweig.

\section{Im 19. Jahrhundert kamen Mittel gegen die «Gefahr der Korpulenz», zerrüttete Nerven, Schwäche oder Müdigkeit auf.}

Auf der individuellen Ebene lässt sich verfolgen, wie Ärzte ihr Behandlungsspektrum mit Enhancementangeboten auszuweiten versuchten, zugleich aber heftig gegen analoge Angebote der nichtärztlichen Konkurrenz ankämpften [5]. Medizinische Publikationen des 18. Jahrhunderts führten zum Beispiel Bleichmittel für einen hellen Hautteint gleichermassen als sinnvolle Rezepturen wie als Beispiele für todbringende Quacksalbereien an. Auch Werke wie Christoph Wilhelm Hufelands «Makrobiotik oder Die Kunst, das menschliche Leben zu verlängern» aus dem Jahr 1797 zeugen letztlich von einem Verständnis des ärztlichen Aufgabenbereichs, der sich nicht auf Linderung und Heilung beschränkt, sondern der Nachfrage nach entsprechenden Angeboten nachkam: Wünsche, das Leben zu verlängern, den Körper zu verjüngen, die Erscheinung zu verschönern oder Fähigkeiten zu verbessern.

Im 19. Jahrhundert kamen Mittel gegen die «Gefahr der Korpulenz», zerrüttete Nerven, Schwäche oder Müdigkeit auf. Hüft-, Kopf- und Nasenformer wurden in Zeitschriften angepriesen. Sie fanden in der ersten Hälfte des 20.Jahrhunderts regen Absatz, vor allem wenn es darum ging, angeblich minderwertige Rassenmerkmale zu kaschieren. Doch nicht nur die äusserliche Form sollte den Idealbildern entsprechen. Auch die Leistungsfähigkeit musste im Rahmen der Industrialisierung auf ein ideales Höchstmass getrimmt sein, wovon vorerst in erster Linie das männliche Geschlecht betroffen war.

Mit Hodenextrakten versuchten Ärzte, die «Lebenskraft» zu erhöhen, und entwickelten im frühen 20.Jahrhundert Verjüngungskuren, die dem neuen Experimentierfeld der Endokrinologie und Trans- plantationsmedizin entsprangen [6]. Obschon solche Anbieter eine akrobatische Gratwanderung unternehmen mussten, um sich vom Vorwurf der Scharlatanerie zu distanzieren, fanden sie doch immer wieder den Beifall hochangesehener, akademischer Mediziner. Die bereits in den 1930er Jahren entwickelte Frischzellentherapie des Schweizer Arztes Paul Niehans, eines angestammten Viszeralchirurgen, wurde in gutbetuchten Kreisen aber bezeichnenderweise erst in der Nachkriegszeit vermehrt genutzt. Zumindest vorübergehend erlangte Niehans einen derart guten Ruf, dass er - als Nachfolger des Penicillinentdeckers Alexander Fleming - sogar in die päpstliche Akademie der Wissenschaften aufgenommen wurde [7].

Wo aber hört Medizin als heilende oder lindernde Tätigkeit auf, wo geht sie in ein Marktangebot über, das auf Bedürfnisse einer Kundschaft eingeht, die nicht primär als Patienten definiert werden können? Diese Frage stellt sich nach wie vor, ob es nun um die Frischzellentherapie des Arztes Niehans oder die Verschreibung von Viagra geht [8].

Ärzte sollten physisches und psychisches Leid heilen oder lindern, mit der aufkommenden Präventivmedizin aber auch möglichst verhindern. Da im Verlauf der Medikalisierung mehr und mehr Normabweichungen als potentielle Gefahren für die Entstehung von physischem und psychischem Leid galten, gehörte ihre Bekämpfung auch zum Aufgabenbereich der Medizin. Sie bedrohten durch physische Risiken wie erhöhte Morbidität und verminderte Lebenserwartung. Sie bedrohten aber auch durch psychische Risiken wie Ausschluss und Vereinsamung. Die Bemühung, die eigene Person körperlich und psychisch $\mathrm{zu}$ verbessern, lässt sich demnach direkt mit der $\mathrm{Ab}$ wendung empfundenen Leids oder potentieller Bedrohungen verknüpfen. Die entsprechenden Enhancementangebote der Medizin dienten in dieser Lesart genauso der Verminderung oder Verhinderung von Leid wie die als seriös geltenden klassisch-therapeutischen Angebote. Eine scharfe und eindeutige Grenzziehung gestaltet sich daher problematisch.

\section{Verinnerlichung von Idealen}

Das Gegenmodell eines leistungsfähigen, sportlichen, jungen und schlanken Ideals entstand aber nicht einfach aufgrund eines äusseren Machteinflusses, der die gesellschaftlichen Werte zu beeinflussen vermochte. Es entsprach einer Moral, die in der ganzen Bevölkerung, mächtig oder machtlos, vorherrschte. Durch die medizinische Propagierung erlangte sie allerdings ein zusätzliches Überzeugungspotential und konnte umso williger im Sinne von Selbsttechniken von den einzelnen Individuen übernommen werden $[9,10$, 11]. Die Praktiken des Einzelnen spiegeln damit über die individuellen Vorstellungen von Gesundheit hinaus den soziokulturellen Umgang mit Normabweichungen wider [12].

Die Vorstellungen von Gesundheit und Schönheit verändern sich auch mit den ökonomischen Bedingungen. In Zeiten des Wirtschaftsaufschwungs re- 
präsentierten Models wie Twiggy ein zerbrechliches, mädchenhaftes Frauenbild. In den 1990er Jahren sollte der ideale Frauenkörper jugendlich muskulös, durchtrainiert und leistungsfähig sein. Der väterlich beschützende Männertyp verlor in dieser Zeit an Prestige, die Haarpracht verschwand, die Muskeln zeigten sich durch die glattrasierte Haut. Entsprechend boomen seither die Fitnesszentren, und der Anabolikakonsum blieb nicht mehr dem Spitzensport vorbehalten. Die sedierende Protestdroge Heroin - um ein weiteres Beispiel zu nennen - wich mehr und mehr anderen aufputschenden Drogen wie Kokain, mit denen eine «normale» Berufstätigkeit weiterhin möglich ist.

\section{Meist entspricht die Nachfrage nach Enhancement- angeboten dem Wunsch nach sozialer Anerkennung.}

Mit der Verinnerlichung gesellschaftlicher Werte wird es schwierig, den individuellen Wunsch einer einzelnen Persönlichkeit zu gewichten. Will sich eine Person vielleicht nur deshalb einem Enhancementeingriff unterziehen, weil sie von äusseren Vorstellungen geleitet wird? Oder ist dieser «Einfluss» von Diskursen nicht stets auch als eine aktive persönliche Interpretation zu werten, die letztlich die individuelle Überzeugung mit bedingt [13]?

Wie kann sich angesichts eines wachsenden Kollektivdrucks auf das Erscheinungsbild die Individualität erhalten? In reicheren Ländern und vor allem in höheren Einkommensschichten lassen sich Normierungsvorstellungen dank medizinischer Eingriffe realisieren. Weisse, regelmässige Zähne, faltenarme Gesichter, straffe Brüste, muskulöse Oberarme und

\section{Was als Bedrohung der Gesundheit verstanden wird, unterliegt dem historischen Wandel.}

schlanke Taillen zeigen nicht nur «Schönheit» und Leistungsfähigkeit, sondern zunehmend auch die Zugehörigkeit zu einer einkommensstarken Bevölkerungsschicht im Sinne einer Distinktion an. Die Frage nach der Zugänglichkeit zu Angeboten des Human Enhancements muss daher diese gesellschaftspolitische und sozialethische Dimension zwingend mit einbeziehen.

In der Regel entspricht die Nachfrage nach Enhancementangeboten dem Wunsch nach sozialer Anerkennung. Die Bemühungen, dunkle Haut auf- zuhellen, krumme Nasen zu begradigen oder Fett zu beseitigen, weisen auf eine Orientierung an entsprechenden gesellschaftlichen Werten hin. Sogar wenn dahinter nur zu deutlich die Hierarchisierung und Diskriminierung von Individuen aufgrund deren äusseren Erscheinungsbildes steht, was heute als «Lookism» untersucht wird, so dienen diese Praktiken letztlich dennoch der individuellen Verringerung von Leid. Ihre Beurteilung muss diese problematische Spannung berücksichtigen, ohne vorschnell zu verurteilen.

\section{Fazit}

- Die historische Analyse bestätigt, dass Krankheit als soziokulturelles, durchaus interessengeleitetes Konstrukt zu verstehen ist. Eine eindeutige, «objektive» Grenzziehung zwischen Therapie und Prävention einerseits und Enhancement andererseits ist nicht möglich. Was als Bedrohung der Gesundheit verstanden wird, unterliegt dem historischen Wandel; dabei hat sich gezeigt, dass bestimmte Auffassungen, «Gesundheit» zu erhalten oder $\mathrm{zu}$ maximieren, fatale Folgen für betroffene Individuen sowie die Gesellschaft haben können.

- Ebenso wandelt sich, was als relevante (Gesundheits-)Norm verstanden wird; an diesem Prozess ist auch die Ärzteschaft aktiv beteiligt. Gesellschaftlich definierte, idealisierte Normvorstellungen lassen sich, sobald sie verinnerlicht sind, nur schwer von individuellen Präferenzen unterscheiden. Wahrgenommene Abweichungen vom Idealbild können zu erheblichem Leidensdruck führen.

- Mit der zunehmenden Medikalisierung wurden Ärzte auch für Aspekte des Alltags (Ernährung, Aussehen, Lebensgewohnheiten usw.) zuständig. Spätestens seit dem 19. Jahrhundert weist die Medizin Enhancementangebote in ihrer Praxis auf und reklamiert damit auch in diesem Bereich Expertise. Versuche, eine echte, «seriöse» Medizin strikt von marktorientierten Enhancementpraktiken abzugrenzen, mögen bisweilen auch von berufspolitischen und ökonomischen Interessen geprägt sein.

- Die historisch gewachsene Zuständigkeit und Expertise der Ärzteschaft, die viele Bereiche des täglichen Lebens berührt, bringt auch eine erhebliche Verantwortung mit sich. So ist bei klinischen Entscheidungen nicht nur der individuelle Patient zu berücksichtigen, sondern es sind auch die Auswirkungen auf das öffentliche Wohl mit zu bedenken, sei es mit Blick auf das beschränkte öffentliche Budget oder auf problematische gesellschaftliche Entwicklungen (z.B. übersteigerte Anforderungen an Leistung oder Verfügbarkeit). 


\section{Literatur}

1 Wiesing U. The History of Medical Enhancement: From Restitutio ad Integrum to Transformatio ad Optimum? In: Gordjin B, Chadwick R (eds.). Medical Enhancement and Posthumanity. The International Library of Ethics, Law and Technology 2. Heidelberg: Springer; 2008: 9-24, hier 9.

2 Wahrig B, Sohn W (Hrsg.). Zwischen Aufklärung, Policey und Verwaltung: Zur Genese des Medizinalwesens 1750-1850. Wiesbaden: Harrassowitz; 2003.

3 Schweizerische Akademie der Medizinischen Wissenschaften (SAMW) in Zusammenarbeit mit Experten und Expertinnen der Verbindung der Schweizer Ärztinnen und Ärzte (FMH) und der Medizinischen Fakultäten. Projekt «Zukunft Medizin Schweiz»: Ziele und Aufgaben der Medizin zu Beginn des 21. Jahrhunderts. Basel; 2004: 30.

4 Stolberg M. Die Harnschau: Eine Kultur- und Alltagsgeschichte. Böhlau: Köln; 2009: v. a. 213-23.

5 Sander S. Der Arzt und die Kosmetik - Zerrbilder im Spiegel der deutschen Medizinhistoriographie. Medizinhist J. 2002;37: 265-300.

6 Schlich T. Die Erfindung der Organtransplantation: Erfolg und Scheitern des chirurgischen Organersatzes 1880-1930. Frankfurt a. M.: Campus; 1998. S. 153-77.
7 Hofer HG. Frischzellen-Fama: Paul Niehans und die westdeutsche Aufbaugesellschaft der 1950er Jahre. In: Eschenbruch N, Balz V, Klöppel U, Hulverscheidt M (Hrsg.). Arzneimittel des 20. Jahrhunderts: Historische Skizzen von Lebertran bis Contergan. Science Studies. Bielefeld: Transcript; 2009: 229-53.

8 Werner MH. Viagra - rechtliche und ethische Fragen. Ärzteblatt Baden-Württemberg. 2002; 81(10): 1-4.

9 Möhring M. Die Regierung der Körper «Gouvernementalität» und «Techniken des Selbst»: Zeithistorische Forschungen/Studies in Contemporary History. Online-Ausgabe. 2006; 2: 3.

10 Maasen S. Schönheitschirurgie: Schnittflächen flexiblen Selbstmanagements. In: Orland B (Hrsg.) Artifizielle Körper. Zürich: Chronos; 2005: 239-59

11 Lengwiler M, Madarász J (Hrsg.). Das präventive Selbst: Eine Kulturgeschichte moderner Gesundheitspolitik. Bielefeld: Transcript; 2010.

12 Gilman S. Making the Body Beautiful: A Cultural History of Aesthetic Surgery. Princeton: Princeton University Press; 1999.

13 Villa P-I. Wider die Rede vom Äusserlichen. In: Villa P-I (Hrsg.). Schön normal: Manipulationen als Technologien des Selbst. Bielefeld: transcript; 2008: 7-18. 